\title{
Using physical therapy to treat temporomandibular disorders. A cohort study
}

\author{
Khaled YA, Brennan MI, Napeñas JO, Quach JE \\ Marquette University, School of Dentistry, USA \\ Correspondence: Khaled YA, Marquette University, School of Dentistry, USA, Email yasser.khaled@marquette.edu
}

Received: May 31, 2018 | Published: June 25, 2018

Copyright@ 2018 Khaled et al. This is an open access article distributed under the terms of the Creative Commons Attribution License, which permits unrestricted use, distribution, and reproduction in any medium, provided the original author and source are credited.

\begin{abstract}
Aim of investigation: Primary aim of this study is to evaluate the results after physical therapy (PT) is prescribed for TMD patients.

Methods: In this retrospective cohort study, we screened 1331 patient records from March 2010 to March 2015 at an oral medicine/orofacial pain practice. Eligible patients were those who were prescribed PT and: had follow up visits within 3 months; were greater than 12years old; had no autoimmune polyarthritis; no history of trauma within 1month of presentation and; no prior experience with PT for this condition. Comparisons were made between two groups: 1) those who underwent PT ( $\mathrm{n}=44)(\mathrm{PT})$ and; 2$)$ those who did not $(\mathrm{n}=44)$ (non-PT). Data was extracted from patient record review. Primary outcome variables included patient reported pain severity scores on a 0-10 scale, and other clinical findings upon examination.

Results: At baseline, non-PT patients had higher levels of distress $(\mathrm{p}=0.0094)$, and less have had oral appliance therapy ( $\mathrm{p}=0.0053)$. At first patient follow up visit, PT patients had lower "current" ( $\mathrm{p}=0.0030)$, "average" $(\mathrm{p}=0.0030)$, and "worst" levels of pain $(\mathrm{p}=0.0003)$, and more subjective improvement of their condition $(\mathrm{p}=0.0157)$. There were no differences between the two groups in clinical findings of tenderness to palpation of TMJs, and measurements of jaw opening and lateral movements.
\end{abstract}

Conclusions: The data from this study supports PT as an effective treatment modality for reducing subjective TMD pain. PT is inexpensive and presents low risk for patient health, making it a cheaper, noninvasive alternative for other modalities such as surgery.

Keywords: physical therapy, bio-mechanical, biological, anatomical, masticatory muscles

\section{Abbreviations}

TMD, temporomandibular disorder; TMJ, temporomandibular joint; PT, physical therapy; $\mathrm{CCOH}$, carolinas center for oral health; TENS. transcutaneous electrical nerve stimulation

\section{Introduction}

Temporomandibular disorder (TMD) is a collective term for structural and functional disorders involving the temporomandibular joint (TMJ) and/or the masticatory muscles, muscles of the head and neck and contiguous tissue components. ${ }^{1}$ Biological, anatomical, biomechanical, behavioral, environmental and/or emotional factors affect the masticatory system, contributing to the development of signs and symptoms and/or perpetuation of TMD. ${ }^{2}$ Therefore, TMD can be considered a multifactorial disease entity. It is mainly characterized by pain and restricted jaw movement, with pain being the most common symptom and the most frequent reason for seeking treatment. ${ }^{3}$ In the literature, the treatments available for TMD include pharmacological management, oral appliances, occlusal equilibration, physical therapy, TMJ surgery, bio-behavioral treatments and patient education. ${ }^{4}$

Physical therapy (PT) for TMD includes self-care treatment, patient education, lifestyle modification and self-awareness about the aggravating factors. ${ }^{5}$ Specifically, it involves active jaw movements, stretching exercises and correction of body and head posture. It is relatively simple, incurs little cost compared with other treatments and ensures the active involvement of patients. Physical therapy has also been shown to provide relief of masticatory muscle and joint pain. ${ }^{6}$ PT for TMD includes TMJ mobilization, soft tissue mobilization of painful muscles, active or passive muscle stretching exercises, gentle isometric tension exercises against resistance and guided opening and closing jaw movements. ${ }^{7}$ These relatively reversible, noninvasive treatments are intended to decrease muscle spasm alter jaw opening-closing patterns and improve coordination of the muscles of mastication. Various reports have suggested that PT is a viable and useful approach towards the management of TMD. ${ }^{8}$

A systematic review by Medlicott and Harris (2006) evaluated the literature on the efficacy of physical therapy interventions for TMD patients and specifically reported the value of a combined approach of active exercises and relaxation techniques. This review favored the use of multifaceted TMD treatments. ${ }^{9}$ A second review on the efficacy of physical therapy for TMD patients found that postural training and exercise demonstrated significant benefits. The authors concluded that active, passive and postural exercises are effective interventions for decreasing the symptoms associated with TMD. ${ }^{10}$

Past literature has uncovered some of the efficacy of PT on TMD; however, no reported studies have documented in totality TMD patient records, which would include other modalities such as pharmacotherapy (e.g.:NSAID's, muscle relaxants, antidepressants), intramuscular therapy (i.e.: trigger point injections, dry needling and botulinum injections), self-measures, and oral appliances on the 
prognosis of TMD patients. Therefore, this study aims to determine the effectiveness of PT on TMD patients, among other comprehensive treatment modalities. ${ }^{11-14}$

\section{Materials and methods}

\section{Population}

This is a retrospective cross-sectional study collecting data from up to 3000 patients. We will be collecting information from patients seen at our outpatient oral medicine clinic, Carolinas Center for Oral Health $(\mathrm{CCOH})$ between March 2010 to March 2015. Appropriate confidentiality agreements and data use/sharing agreements will be in place prior to any information sharing. Appropriate Ethics Board and Institutional Review Board approval will be obtained for this retrospective analysis. The study is conducted in accordance with the principles of the Declaration of Helsinki.

\section{Inclusion criteria for patients}

i. Patients with a diagnosis of temporomandibular disorder on initial examination and/or follow up to include the following specific diagnoses: disc displacement (with or w/o) reduction, retrodiscitis, ankyloses, open or closed fracture, synovitis and capsulitis, primary or secondary osteoarthritis (degenerative joint disease), TMJ adhesions, dislocation, temporal tendonitis, local myalgia, myofascial pain, and centrally mediated myalgia

ii. 12 yrs. or older

iii. Follow up appointments within 3 months from the date the physical therapy was prescribed.

\section{Exclusion criteria for patients}

i. Trauma patients presenting to the clinic within 1 month after trauma.

ii. Patients with systemic polyarthrides.

\section{Research plan and study variables}

The databases will be reviewed from the electronic medical records for all the patients that were seen in $\mathrm{CCOH}$ between March 2010 and March 2015. The following variables will be collected for all patients (Appendix): age, gender, medications (at initial visit), concurrent relevant systemic problems, social history (i.e.: smoking, current alcohol use), vitals (i.e.: heart rate, systolic and diastolic blood pressure), quality of pain, severity of pain, pain stimulating factors, frequency and duration of pain, previous treatments (i.e.: previous providers, previous treatment modalities). All concurrent medications can be either collected from a questionnaire completed at the time of the visit by the patient or via a confirmation through electronic medical records. Then, they will be either sorted into categories or inserted singularly.

We will collect data $(\mathrm{Y} / \mathrm{N})$ on the following pertinent medical history: depression, anxiety, eating disorder (anorexia and bulimia), history of trauma, Arthritis (osteoarthritis), back and shoulder pain and autoimmune disorders. With respect to complaints pertaining to TMD, we will collect data on previous complaint or history of: disc displacement (with or w/o) reduction, retrodiscitis, ankyloses, open or closed fracture, synovitis and capsulitis, systemic polyarthritides, rheumatoid arthritis, primary or secondary osteoarthritis, TMJ adhesions, dislocation and temporal tendonitis. At the time of visit, all patients were examined clinically to determine a diagnosis. We do not anticipate problems with enrollment and data collection to complete this retrospective study.

\section{Statistical analysis}

Univariate analysis will include chi square test (dichotomous variables) or t-test (continuous variables). Non-parametric tests including the Fisher's exact test and Wilcoxon rank-sum test will be used if when appropriate. Predictor variables will be selected by univariate analysis $(p<0.1)$ or factors determined to be essential for a multivariate analysis (e.g. age and gender). Analyses will be carried out using the SAS statistical program (SAS Institute Inc., Cary, North Carolina, USA). Physical therapy (PT) is among the widely-used treatment modalities to address temporomandibular disorders (TMD). PT is a conservative, cheaper alternative to other treatments and presents lower risk because it is non-invasive unlike high risk procedures such as surgery. However, there is very limited data looking at outcomes in this patient cohort. The aim of this study is to evaluate the outcomes after PT is prescribed for patients with TMD. ${ }^{15-18}$

Temporomandibular disorders (TMDs), also referred to as craniomandibular disorders, consist of a group of pathologies affecting the masticatory muscles, the temporomandibular joint (TMJ), and related structures. TMD is considered a musculoskeletal disorder of the masticatory system that affects more than $25 \%$ of the general population. Temporomandibular disorder is usually manifested by one or more of the following signs or symptoms: pain, joint sounds, limitation in jaw movement, muscle tenderness, and joint tenderness. ${ }^{19-22}$ Patients with chronic TMD frequently report symptoms of depression, poor sleep quality, and low energy. It also is commonly associated with other symptoms affecting the head and neck region such as headache, ear-related symptoms, and cervical spine disorders. Chronic TMD can also interfere with normal social activity and interpersonal relationships and negatively affect the ability to maintain employment. ${ }^{23-28}$

The American Academy of Craniomandibular Disorders and the Minnesota Dental Association have confirmed that physical therapy is an important treatment for TMDs. Physical therapy is intended to relieve musculoskeletal pain, reduce inflammation, and restore oral motor function. Lots of physical therapy interventions are very effective in managing TMD, including electrophysical modalities, exercise, and manual therapy techniques. ${ }^{29}$ Electrophysical modalities include ultrasound, microwave, laser, and transcutaneous electrical nerve stimulation (TENS). Physical therapy interventions often include therapeutic exercises for the masticatory or cervical spine muscles to improve strength and mobility in the region. Manual therapy techniques are commonly used to reduce pain and restore mobility. ${ }^{30-32}$ Oral exercise devices, such as the Therabite Jaw Motion Rehabilitation System, are mechanical aids that provide passive stretch to the TMJ to improve mandibular range of motion. Physical therapy interventions also may include, or focus on, associated impairments of the craniocervical system such as poor posture, cervical muscle spasm, cervical pain, or referred pain from the cervical spine. Acupuncture is considered a specialty field within the scope of practice for many physical therapists working in countries such as Canada, the United Kingdom, and Australia. ${ }^{33}$ Management of TMD, however, most often involves a multidisciplinary approach. Dentists, orthodontists, psychologists, physical therapists, and physicians 
work together to address the condition of the patient with TMD. ${ }^{34-38}$ Conservative treatment is usually the treatment of choice because the symptomatology of the condition often is improved by use of occlusal splints, physical therapy, medication, and orthodontic treatment. Many reviews have been published on conservative treatments, often recommending a multidisciplinary treatment approach for TMD; however, research evidence supporting this approach is usually not provided. ${ }^{39}$

More recently, there has been an interest in the relative effectiveness of specific conservative interventions for TMD, and, as a result, a number of systematic reviews have been performed in the area. One systematic review by Ernst and White, published in 1999, examined the efficacy of acupuncture for TMD. Based on preliminary findings from only 3 trials in the area, the authors concluded that, although acupuncture may be a potentially effective intervention for TMD, more rigorous trials were needed to confirm this conclusion. A meta-analysis, also published in 1999, examined the efficacy of electromyographic (EMG) biofeedback for TMD. Based on their meta-analysis of 13 studies, the authors concluded that there was evidence to support the use of EMG biofeedback in the management of TMD. The conclusions of the meta-analysis, however, were based on data from controlled and uncontrolled trials, and the findings therefore should be interpreted with caution. ${ }^{40-42}$ More recently, 2 separate systematic reviews have examined the effectiveness of stabilization splint therapy (2004) and occlusal adjustment (2005)17 in the management of TMD. Based on the findings of these 2reviews, there is currently insufficient evidence to support or refute the use of stabilization splint therapy and no evidence to support occlusal adjustment in the management of TMD.

To date, the question of whether physical therapy interventions are effective in the management of TMD remains unanswered. Thus, the purpose of this study was to evaluate the effectiveness of physical therapy interventions in the management of TMD. ${ }^{43}$ This was a single center, retrospective, observational study. We screened patient records of those who presented to our outpatient oral medicine practice between March 2010 to March 2015 with symptoms of TMD. Eligible patients were: those who were diagnosed with a TMD (e.g.: synovitis/ capsulitis/arthralgia of TMJ, articular disc disorder (reducing or nonreducing), myofascial pain) were prescribed to have PT as part of their treatment plan; had follow up visits within 3months of the initial visit; were greater than 12years old; had no autoimmune polyarthritides; no history of trauma within 1 month of presentation and; no prior experience with PT for this condition. Comparisons were made between two groups: 1) involving patients who were prescribed PT and attended PT by the first follow up (1st FU) visit (PT group) and; 2) patients who were prescribed PT but did not attend PT by the $1^{\text {st }}$ FU (non-PT group). Information gathered included demographics, medical history, social history, and baseline and follow up information from history and clinical examination. Primary outcome variables included patient reported pain severity scores on a $0-10$ scale (to include "current," "average," and "worst" levels of pain,) and other clinical findings upon examination to include maximum interincisal mouth opening with and without pain (in millimeters) (Figure 1). ${ }^{44}$

\section{Results}

Within that 5-year span, there were 1331 patients seen for TMD symptoms. After screening of records, 298patients met inclusion criteria and were divided into the two groups in the following manner:
1) PT group ( $n=228)$ and; 2$)$ non-PT group $(n=70)$. There was no difference in mean age \pm SD between the two groups: $\mathrm{PT} 44.4 \mathrm{yrs} \pm 16.98$ vs. non-PT 46.3yrs $\pm 16.2(\mathrm{p}=0.32$ ). A significant difference was found in the proportion of men vs. women who went to PT. Out of the 50 men included in the study, $76 \%$ went to PT and $24 \%$ did not; this is compared to $56 \%$ of women who went for PT vs. $44 \%$ who did not $(\mathrm{p}=0.0085)$.

Demographics
Medical and Social History
PT?
Treatment Modalities
Mouth Movements (mm)
Initial and Follow-up Level of Pain
- 0 to 10 scale

\section{Figure I Statistical analysis}

There was a statistically significant difference between PT and nonPT groups in mean reduction of reported average, and worst pain levels between the initial and 1 st FU visits. Mean reduction \pm SD of average pain levels were: PT $1.65 \pm 2.33$ and; non-PT $0.90 \pm 2.59$ $(\mathrm{p}=0.04)$. Mean reduction of worst pain levels were: PT $1.84 \pm 2.52$ and non-PT $1.04 \pm 2.60(\mathrm{p}=0.02)$. Mean reduction of current pain did not have any statistically significant differences between the two groups:PT $0.98 \pm 2.65$ and non-PT $0.52 \pm 2.42(\mathrm{p}=0.13)$. There was also a statistically significant difference between groups in changes in maximum mouth opening with pain and without pain. Mean difference in maximum mouth opening with pain was PT $-2.27 \pm 6.25$ and nonPT $0.64 \pm 5.55(\mathrm{p}=0.02)$. Mean difference in maximum mouth opening without pain was PT-5.50 \pm 7.53 and non-PT-2.3 $\pm 6.48(\mathrm{p}=0.0005)$. There were no differences between the two groups in clinical findings of tenderness to palpation of TMJs and muscles of mastication, and lateral range of movements (Figure 2-5).

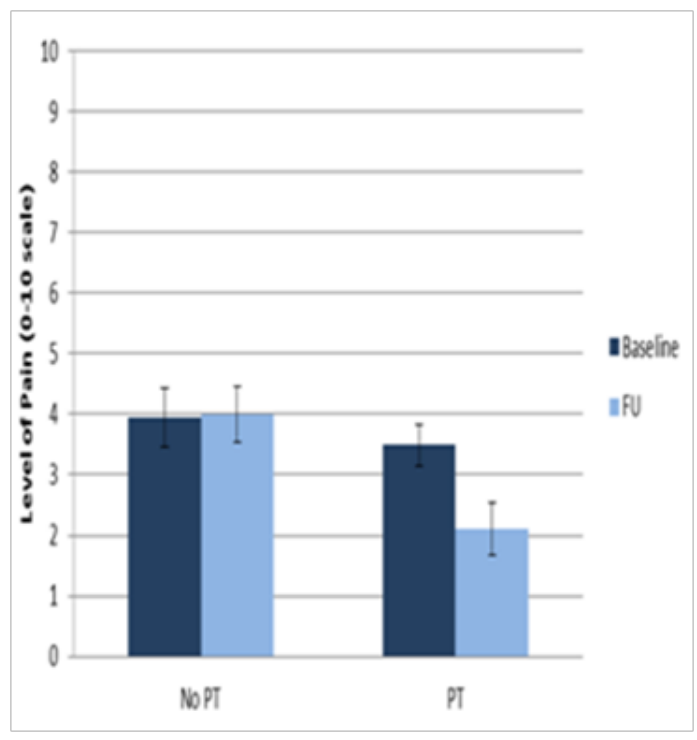

Figure 2 Current level of pain
Submit your Article | www.ologypress.com/submit-article

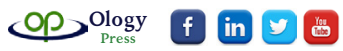

Citation: Khaled YA, Brennan MI, Napeñas JO, et al. Using physical therapy to treat temporomandibular disorders. A cohort study.J Dent Maxillofacial Res. (20I8); I (I):3I-35. DOI: I0.3088I/jdsomr.00008 


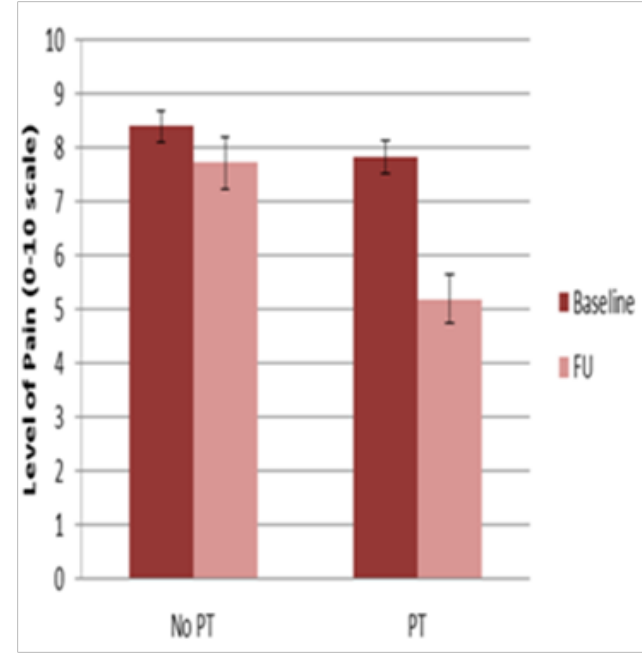

Figure 3 Worst level of pain

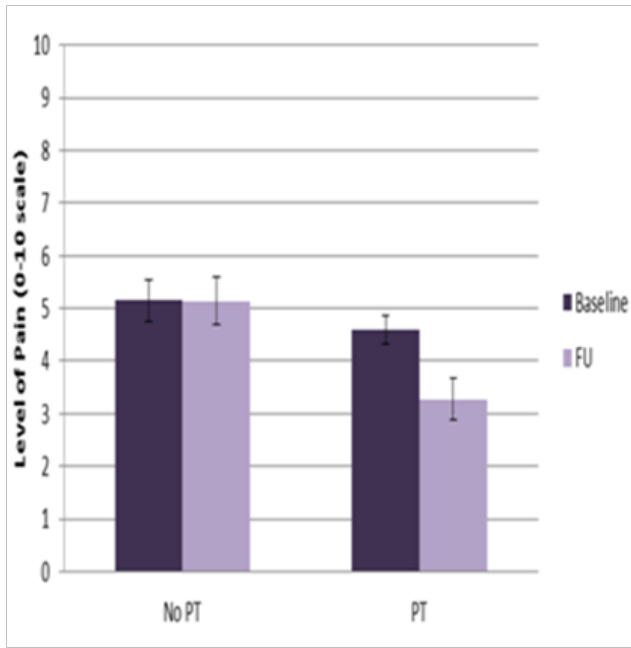

Figure 4 Average level of pain

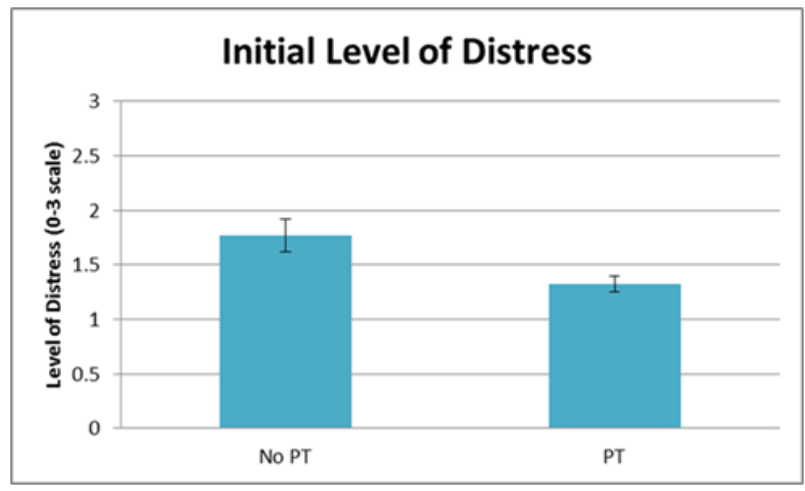

Figure 5 Initial level of distress

\section{Conclusion}

TMD patients who proceeded to PT had greater improvements in reported average and worst pain, and maximum range of mouth opening than those who did not undergo PT. The results of our study supports PT as an effective measure to treat TMD. Although we were able to derive significant data from this study, there were limitations. Because this was a retrospective study, we encountered some missing data in the patient records due to variability in clinical notes over the 5 -year span. In addition, comparisons between various specific physical therapy modalities were not taken into consideration. Additionally, as this study was not randomized, selection bias may be present

\section{Acknowledgements}

None.

\section{Conflict of interest}

The author declares no conflict of interest.

\section{References}

1. Okeson JP. Guidelines for assessment, diagnosis and management. Chicago: Quintessence Publishing Co; 1996. p. 113-184.

2. De Leeuw R. American Academy of Orofacial Pain: Guidelines for assessment, diagnosis, and management. 4th ed. Chicago: Quintessence Publishing; 2008. p. 131-141.

3. Dworkin SF, Huggins KH, Leresche L, et al. Epidemiology of signs and symptoms in temporomandibular disorders: clinical signs in cases and controls. J Am Dent Assoc. 1990;120(3):273-281.

4. Wright A, Sluka KA. Nonpharmacological treatments for musculoskeletal pain. Clin J Pain. 2001;17(1):33-46.

5. Okeson JP. Management of temporomandibular disorders and occlusion. 5th ed. St Louis: Mosby; 2003. p. 371-393.

6. Michelotti A, Stinks MH, Ferrule M, et al. The additional value of a home physical therapy regimen versus patient education only for the treatment of myofascial pain of the jaw muscles: short-term results of a randomized clinical trial. J Orofac Pain. 2004;18(2):114-125.

7. Hanten WP, Olson SL, Butts NL, et al. Effectiveness of a home program of ischemic pressure followed by sustained stretch for treatment of myofascial trigger points. Phys Ther. 2000;80(10):997-1003.

8. Michelotti A, de Wijer A, Stinks M, et al. Home-exercise regimes for the management of non-specific temporomandibular disorders. J Oral Rehabil. 2005;32(11):779-785.

9. Rocabado M, Iglarsh Z. Musculoskeletal approach to maxillofacial pain. Philadelphia: JB Lippincott; 1991.

10. Von Piekartz H. Kiefer, gesichts- und zervikalregion. Kraniomandibulare Region-klinische Muster und Management Thieme Verlag, Stuttgart; 2005. p. 168-225.

11. Carmeli E, Sheklow SL, Bloomenfeld I. Comparative study of repositioning splint therapy and passive manual range of motion techniques for anterior displaced temporomandibular disc with unstable excursive reduction. Physiotherapy. 2001;87(1):26-36.

12. Nicolakis P, Erdogmus B, Kopf A, et al. Effectiveness of exercise therapy in patients with internal derangement of the temporomandibular joint. $J$ Oral Rehabil. 2001;28(12):1158-1164.

13. Kalamir A, Pollard H, Vitiello AL, et al. Manual therapy for temporomandibular disorders: a review of the literature. J Body Mov Ther. 2007;11(1):84-90.

14. Medlicott MS, Harris SR. A systematic review of the effectiveness of exercise, manual therapy, electrotherapy, relaxation training, and biofeedback in the management of temporomandibular disorder. Phys Ther. 2006;86(7):955-973. 
15. McNeill C. Epidemiology. In: McNeil C, et al. editors. Temporomandibular disorders: guidelines for classification, assessment, and management. 2nd ed. Chicago Ill: Quintessence Publishing Co; 1993. p. 19-22.

16. Di Fabio RP. Physical therapy for patients with TMD: a descriptive study of treatment, disability, and health status. J Orofac Pain. 1998;12(2):124135 .

17. Gremillion HA. The prevalence and etiology of temporomandibular disorders and orofacial pain. Tex Dent J. 2000;117(7):30-39.

18. Benoit P. History and physical examination for TMD. In: Kraus SL, et al, editor. Temporomandibular Disorders. 2nd ed. New York, NY: Churchill Livingstone; 1994. p. 71-98.

19. De Wijer A, de Leeuw JR, Steenks MH, Bosman F. Temporomandibular and cervical spine disorders: self-reported signs and symptoms. Spine. 1996;21(14):1638-1646.

20. Morris S, Benjamin S, Gray R, et al. Physical, psychiatric and social characteristics of the temporomandibular disorder pain dysfunction syndrome: the relationship of mental disorders to presentation. $\mathrm{Br}$ Dent J. 1997;182(7):255-260.

21. Sturdivant J, Fricton JR. Physical therapy for temporomandibular disorders and orofacial pain. Curr Opin Dent. 1991;1(4):485-496.

22. Rocabado M. The importance of soft tissue mechanics in stability and instability of the cervical spine: a functional diagnosis for treatment planning. Cranio. 1987;5(2):130-138.

23. Fricton JR, Dubner R. Orofacial pain and temporomandibular disorders New York: Raven Press; 1995. 576 p.

24. Feine JS, Lund JP. An assessment of the efficacy of physical therapy and physical modalities for the control of chronic musculoskeletal pain. Pain. 1997;71(1):5-23.

25. Dimitroulis G, Gremillion HA, Dolwick MF, et al. Temporomandibular disorders, 2: non-surgical treatment. Aust Dent J. 1995;40(6):372-376.

26. McNamara JA, Seligman DA, Okeson JP. Occlusion, orthodontic treatment, and temporomandibular disorders: a review. J Orofac Pain. 1995;9(1):73-90.

27. Crider AB, Glaros AG. A meta-analysis of EMG biofeedback treatment of temporomandibular disorders. J Orofac Pain. 1999;13(1):29-37.

28. Ernst E, White AR. Acupuncture as a treatment for temporomandibula joint dysfunction: a systematic review of randomized trials. Arch Otolaryngol Head Neck Surg. 1999;125(3):269-272.

29. Forssell H, Kalso E, Koskela $P$, et al. Occlusal treatments in temporomandibular disorders: a qualitative systematic review of randomized controlled trials. Pain. 1999;83(3):549-560.
30. Al-Ani MZ, Davies SJ, Gray RJ, et al. Stabilisation splint therapy for temporomandibular pain dysfunction syndrome. Cochrane Database Syst Rev. 2004;(1):CD002778

31. Koh H, Robinson PG. Occlusal adjustment for treating and preventing temporomandibular joint disorders. Cochrane Database Syst Rev. 2003;(1):003812.

32. Lohr KN, Carey TS. Assessing "best evidence": issues in grading the quality of studies for systematic review. Jt Comm J Qual Improv. 1999;25(9):470-479.

33. Dickersin K, Scherer R, Lefebvre C. Identifying relevant studies for systematic reviews. BMJ. 1994;309(6964):1286-1291.

34. Landis JR, Koch GG. An application of hierarchical kappa-type statistics in the assessment of majority agreement among multiple observers. Biometrics. 1977;33(2):363-374.

35. Landis JR, Koch GG. The measurement of observer agreement for categorical data. Biometrics. 1977;33(1):159-174.

36. Jadad AR, Moore RA, Carroll D, et al. Assessing the quality of reports of randomized clinical trials: is blinding necessary? Control Clin Trials. 1996;17(1):1-12.

37. Juni P, Altam D, Egger M. Assessing the quality of randomized controlled trials. In: Egger M, et al. editors. Systematic Reviews in Health Care. 3rd ed. London, United Kingdom: BMJ Books; 2003. p. 87-108.

38. Moher D, Jadad AR, Nichol G, et al. Assessing the quality of randomized controlled trials: an annotated bibliography of scales and checklists. Control Clin Trials. 1995;16(1):62-73.

39. de Vet HC, de Bie RA, van der Heijden G, et al. Systematic reviews on the basis of methodological criteria. Physiotherapy. 1997;83(6):284-289.

40. Haywood S. Systematic overview project. Edmonton, Alberta. Canada: Alberta Heritage Foundation for Medical Research; 1997.

41. Magee DJ. Systematic reviews and functional outcomes measurements: A Teaching Manual. Edmonton, Alberta, Canada: University of Alberta, Department of Physical Therapy; 1998.

42. Magee DJ, Oborn-Barret E, Turner S, et al. A systematic overview of the effectiveness of physical therapy intervention on soft tissue neck injury following trauma. Physiother Can. 2000;52(2):111-130.

43. McNeely ML, Torrance G, Magee DJ. A systematic review of physiotherapy for spondylolysis and spondylolisthesis. Man Ther. 2003;8(2):80-91.

44. Colle F, Rannou F, Revel M, et al. Impact of quality scales on levels of evidence inferred from a systematic review of exercise therapy and low back pain. Arch Phys Med Rehabil. 2002;83(12):1745-1752. 